\title{
Correct-by-design Control Synthesis for Multilevel Converters using State Space Decomposition
}

\author{
Gilles Feld \\ SATIE, ENS Cachan \& CNRS, France \\ Denis Labrousse \\ SATIE, ENS Cachan \& CNRS, France
}

\author{
Laurent Fribourg \\ LSV, ENS de Cachan \& CNRS, France \\ Bertrand Revol \\ SATIE, ENS Cachan \& CNRS, France
}

\author{
Romain Soulat \\ LSV, ENS de Cachan \& CNRS, France
}

\begin{abstract}
High-power converters based on elementary switching cells are more and more used in the industry of power electronics owing to various advantages such as lower voltage stress and reduced power loss. However, the complexity of controlling such converters is a major challenge that the power manufacturing industry has to face with. The synthesis of industrial switching controllers relies today on heuristic rules and empiric simulation. The state of the system is not guaranteed to stay within the limits that are admissible for its correct electrical behavior. We show here how to apply a formal method in order to synthesize a correct-by-design control that guarantees that the power converter will always stay within a predefined safe zone of variations for its input parameters. The method is applied in order to synthesize a correct-by-design control for 5-level and 7-level power converters with a flying capacitor topology. We check the validity of our approach by numerical simulations for 5 and 7 levels. We also perform physical experimentations using a prototype built by SATIE laboratory for 5 levels.
\end{abstract}

\section{Introduction}

Switched control has gained much attention recently due to its property of being easily implemented, especially in the field of power converters. Power converters play an important role in the field of renewable energy: they are used to connect renewable sources to powergrids, optimize the efficiency of solar panels and wind generators (see, e.g., [1]). In some topologies, there is however a dramatic increase of the number of switches, which entails an increasing number of degrees of freedom, and complicates the controller design. There is therefore a niche of application for formal methods in order to produce correct-by-design control methods. The general function of a multilevel power converter is to synthesize a desired voltage from several levels of DC voltage. For this reason, multilevel power converters can easily provide the high power required by large electric drive systems. A multilevel converter is a power converter made of capacitors and switching cells (as well as opposite switching cells which are in complementary positions);

In this paper, we consider the design of control policies for power converters with a number of levels $\ell=5$ and $\ell=7$. A multilevel converter for $\ell=5$ is schematized on Figure 1 . According to the positions of the cells, one is able to fraction the load voltage. By controlling the global position of the switches during a simple fixed time-stepping procedure, it is then possible to generate a staircase voltage with levels that approximates a triangular or a sinusoidal waveform (see Figure 2, for 5 levels).

The problem which arises is to select the appropriate switching control strategy among a number of combinations of switch positions which increases exponentially with the number of levels (and pairs of switches). A crucial difficulty comes from the fact that, in order to be admissible, the control of the switching cells must guarantee that the voltages across the cell-capacitors are constrained within a certain

S.-W. Lin and L. Petrucci (Eds.): 2nd French Singaporean Workshop on Formal Methods and Applications EPTCS 156, 2014, pp. 5-16 doi 10.4204/EPTCS.156.5 (c) G. Feld, L. Fribourg, D. Labrousse, B. Revol \& R. Soulat This work is licensed under the Creative Commons Attribution License. 


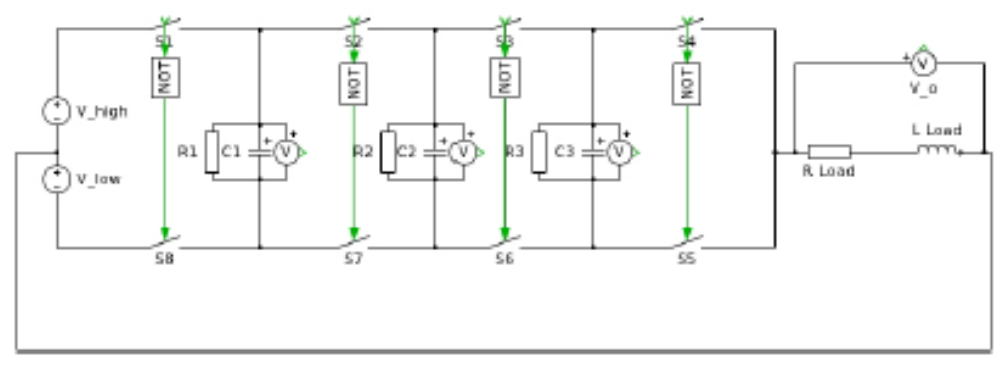

Figure 1: Electrical scheme of a 5-level flying capacitor converter

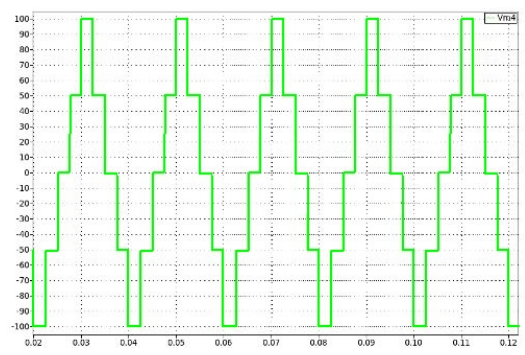

Figure 2: Staircase output voltage waveform for a 5-level converter

range defined by the device blocking voltage rating. The control must thus guarantee a safety property, called "capacitor voltage balancing": the voltage of each individual capacitor should stay inside a limited predefined interval. The synthesis of industrial switching controllers relies today on heuristic rules and empiric simulation. The state of the system is not guaranteed to always satisfy capacitor voltage balancing. In this paper, we show how to synthesize a control, by applying a formal method, called state space decomposition procedure [4]. The synthesized control is "correct-by-design" because it is ensured to make the electrical state parameters of the system stay within predefined safe zones of variations. Numerical simulations, performed at levels $\ell=5,7$, confirm the safety properties of the synthesized control. Physical experimentations are also successfully performed on a prototype built by SATIE Electronics Laboratory, at level $\ell=5$.

\section{Outline of the paper}

In Section 2, we present the principles of the state space decomposition method. In Section 3, we apply the method in order to synthesize the control of multilevel converters with a flying capacitor topology, for 5 and 7 levels. In Section 4, we present physical experimentations done with a prototype of 5-level converter. We conclude in Section 5 .

\section{State Space Decomposition Method}

A multilevel converter can be seen as a "switched system", where the different operating modes depend on the positions of the switching cells. In this section, we describe a general method that is useful for proving properties of switched systems. The method will be subsequently applied to multilevel converters in Section 3 , 


\subsection{Model of affine sampled switched systems}

A switched system $\Sigma$ is defined by a finite family of differential equations of the form $\left\{\dot{x}=f_{u}(x)\right\}_{u \in U}$ where $U$ is a finite set of modes (see, e.g., [5, 14]). In the following, we consider that the dynamics of the subsystems are affine (i.e., $f_{u}(x)$ is of the form $A_{u} x+b_{u}$ with $A_{u} \in \mathbb{R}^{n \times n}$ and $b_{u}$ a vector of $\mathbb{R}^{n}$ ). The control problem for a switched system $\Sigma$ is to find a piecewise constant law $\mathbf{u}: \mathbb{R}_{\geq 0} \rightarrow U$ in order to achieve some pertained goals. The switching instants are the times at which $\mathbf{u}$ changes its value. An affine sampled switched system is a switched system for which the switching instants occur at integer multiples of $\tau$ (called sampling parameter). We will use $\mathbf{x}(t, x, u)$ to denote the point reached by $\Sigma$ at time $t$ under mode $u$ from the initial condition $x$. This gives a transition relation $\rightarrow{ }_{u}^{\tau}$ defined for $x$ and $x^{\prime}$ in $\mathbb{R}^{n}$ by: $x \rightarrow{ }_{u}^{\tau} x^{\prime}$ iff $\mathbf{x}(\tau, x, u)=x^{\prime}$. Given a set $X \subset \mathbb{R}^{n}$, we define:

$$
\operatorname{Post}_{u}(X)=\left\{x^{\prime} \mid x \rightarrow{ }_{u}^{\tau} x^{\prime} \text { for some } x \in X\right\} \text {. }
$$

It can be seen that $\operatorname{Post}_{u}(X)$ is the result of an affine transformation of the form $C_{u} X+d_{u}$ with $C_{u} \in \mathbb{R}^{n \times n}$ and $d_{u}$ a vector of $\mathbb{R}^{n}$.

A pattern $\pi$ is defined as a finite sequence of modes. A $k$-pattern is a pattern of length at most $k$. The mapping Post $\pi$ is itself an affine transformation.

Given a pattern $\pi$ of the form $\left(u_{1} \cdots u_{m}\right)$, and a set $X \subset \mathbb{R}^{n}$, the unfolding of $X$ via $\pi$, denoted by $\operatorname{Unf}_{\pi}(X)$, is the set $\bigcup_{i=0}^{m} X_{i}$ with:

- $X_{0}=X$,

- $X_{i+1}=$ Post $_{u_{i+1}}\left(X_{i}\right)$, for all $0 \leq i \leq m-1$.

The unfolding thus corresponds to the set of all the intermediate states produced when applying pattern $\pi$ to the states of $X$.

\subsection{Safety control problem}

A safety property is typically expressed using a subset $S$ of the continuous state space, called safe set. In a simple formulation, $S$ is a box, i.e., a cartesian product of intervals that specify the minimum and maximum values tolerated for each state component. Given a safe set $S$, and a domain of interest $R \subseteq S$, we can define the notion of "safe control" in this context as follows.

Definition 1 Given a domain of interest $R$ and safe set $S$ with $R \subseteq S$, a safe control of $R$ w.r.t. $S$ is a function that associates to each $x \in R$ a pattern $\pi$ such that:

- $\operatorname{Post}_{\pi}(\{x\}) \subseteq R$, and

- $U n f_{\pi}(\{x\}) \subseteq S$.

Given a domain of interest $R$ and a set $S$ with $R \subseteq S$, the safety control problem consists in finding a safe control of $R$ w.r.t. $S$. In [4], in order to solve such a problem, we introduced the notion of "(safe) decomposition".

Definition 2 Given a set $R \subset \mathbb{R}^{n}$ and a set $S$ with $R \subseteq S$, a safe decomposition of $R$ w.r.t. $S$ is a set $\Delta$ of the form $\left\{V_{i}, \pi_{i}\right\}_{i \in I}$, where I is a finite set of indices, $V_{i}$ s are subsets of $R, \pi_{i} s$ are k-patterns, such that:

- $\bigcup_{i \in I} V_{i}=R$,

- for all $i \in I$ : Post $_{\pi_{i}}\left(V_{i}\right) \subseteq R$, and

- for all $i \in I$ : $U n f_{\pi_{i}}\left(V_{i}\right) \subseteq S$. 
A decomposition $\Delta=\left\{\left(V_{i}, \pi_{i}\right)\right\}_{i \in I}$ naturally induces a state-dependent control on $R$. Furthermore, the controlled trajectories starting from $R$ never leave $S$. Indeed, given a starting state $x_{0}$ in $R$, we know that $x_{0} \in V_{i}$ for some $i \in I$ (since $R=\bigcup_{i \in I} V_{i}$ ); one thus applies $\pi_{i}$ to $x_{0}$, which gives a new state $x_{1}$ that belongs itself to $R$ (since $\operatorname{Post}_{\pi_{i}}\left(V_{i}\right) \subseteq R$ ); furthermore, since $U n f_{\pi_{i}}\left(V_{i}\right) \subseteq S$, all the intermediate states produced by application of $\pi_{i}$ are guaranteed to belong to $S$. The process can then be repeated on $x_{1}$, and so on iteratively. Formally, we have:

Proposition 1 Suppose that $\Delta$ is a safe decomposition of $R$ w.r.t. S. Then the control of $R$ induced by $\Delta i$ s safe w.r.t $S$.

The problem of finding a safety controller thus reduces to the problem of finding a safe decomposition $\Delta$. The latter problem can be solved by using the state space decomposition method [4], as explained below.

\subsection{State space decomposition method}

We give here a simple algorithm, adapted from [4], called Decomposition algorithm. Given a set $R$ and a set $S$ with $R \subseteq S$, the algorithm outputs, when it succeeds, a decomposition $\Delta$ of $R$ w.r.t $S$, of the form $\left\{V_{i}, \pi_{i}\right\}_{i \in I}$. The input sets $R$ and $S$ are given under the form of boxes of $\mathbb{R}^{n}$ (i.e., cartesian products of $n$ closed intervals). The subsets $V_{i}$ s of $R$ are boxes that are obtained by repeated bisection. At the beginning, the Decomposition procedure calls sub-procedure Find_Pattern in order to get a $k$-pattern $\pi$ such that $\operatorname{Post}_{\pi}(R) \subseteq R$ and $U n f_{\pi}(R) \subseteq S$. If it succeeds, then it is done. Otherwise, it divides $R$ into $2^{n}$ sub-boxes $V_{1}, \ldots, V_{2^{n}}$ of equal size. If for each $V_{i}$, Find_Pattern gets a $k$-pattern $\pi_{i}$ such that Post $\pi_{i}\left(V_{i}\right) \subseteq R$ and $U n f_{\pi_{i}}\left(V_{i}\right) \subseteq S$, it is done. If, for some $V_{j}$, no such pattern exists, the procedure is recursively applied to $V_{j}$. It ends with success when a safe decomposition of $R$ w.r.t. $S$ is found, or failure when the maximal degree $d$ of decomposition is reached. The algorithmic form of the procedure is given in Algorithms 1 and 2. (For the sake of simplicity, we consider the case of dimension $n=2$, but the extension to $n>2$ is straightforward.) The main procedure Decomposition $(W, R, S, D, K)$ is called with $R$ as input value for $W$, $d$ for input value for $D$, and $k$ as input value for $K$; it returns either $\left\langle\left\{\left(V_{i}, \pi_{i}\right)\right\}_{i}\right.$, True $\rangle$ with $\bigcup_{i} V_{i}=W$, $\bigcup_{i} \operatorname{Post}_{\pi_{i}}\left(V_{i}\right) \subseteq R, \bigcup_{i} U n f_{\pi_{i}}\left(V_{i}\right) \subseteq S$ or $\left\langle_{-}, F a l s e\right\rangle$. Procedure Find_Pattern $(W, R, S, K)$ looks for a $K$-pattern $\pi$ for which $\operatorname{Post}_{\pi}(W) \subseteq R$ and $U n f_{\pi}(W) \subseteq S$ : it selects all the $K$-patterns by non-decreasing length order until either it finds such a pattern $\pi$ (output: $\langle\pi, T r u e\rangle$ ), or none exists (output: $\left\langle_{-}, F a l s e\right\rangle$ ). The correctness of the procedure is stated as follows.

Theorem 1 If Decomposition $(R, R, S, d, k)$ returns $\langle\Delta$, True $\rangle$, then $\Delta$ is a safe decomposition of $R$ w.r.t. $S$.

In [4], we have developed a tool that implements the Decomposition procedure, using zonotopes [6], and is written in Octave [10]. We now describe the application of this tool, called MINIMATOR [9], for synthesizing controllers of multilevel converters.

\section{Application to Multilevel Converters}

\subsection{Multilevel converters as switched systems}

There are different possible topologies for multilevel power converters: neutral-point clamped, cascaded H-bridge, Modular Multilevel Converter (see e.g., [12, 2, 7, 8]). We focus here on the flying capacitor topology [8]. The electrical scheme of a 5-level converter was given in Figure 11. There are 4 pairs of switching cells $S_{1}, S_{2}, S_{3}, S_{4}$ (the high-side switch conducting position is indicated by 1 and the lowside switch conducting position by 0 ), and 3 capacitors $C_{1}, C_{2}, C_{3}$. The state of the system is $x(t)=\left[\begin{array}{lll}v_{1}(t) & v_{2}(t) & v_{3}(t) i(t)\end{array}\right]^{T}$ where $v_{j}(t)$ is the voltage across $C_{j}(1 \leq j \leq 3)$ and $i(t)$ is the current flowing in the circuit. The duration of a cycle is $T=8 \tau$. The mode of the system is characterized by the 

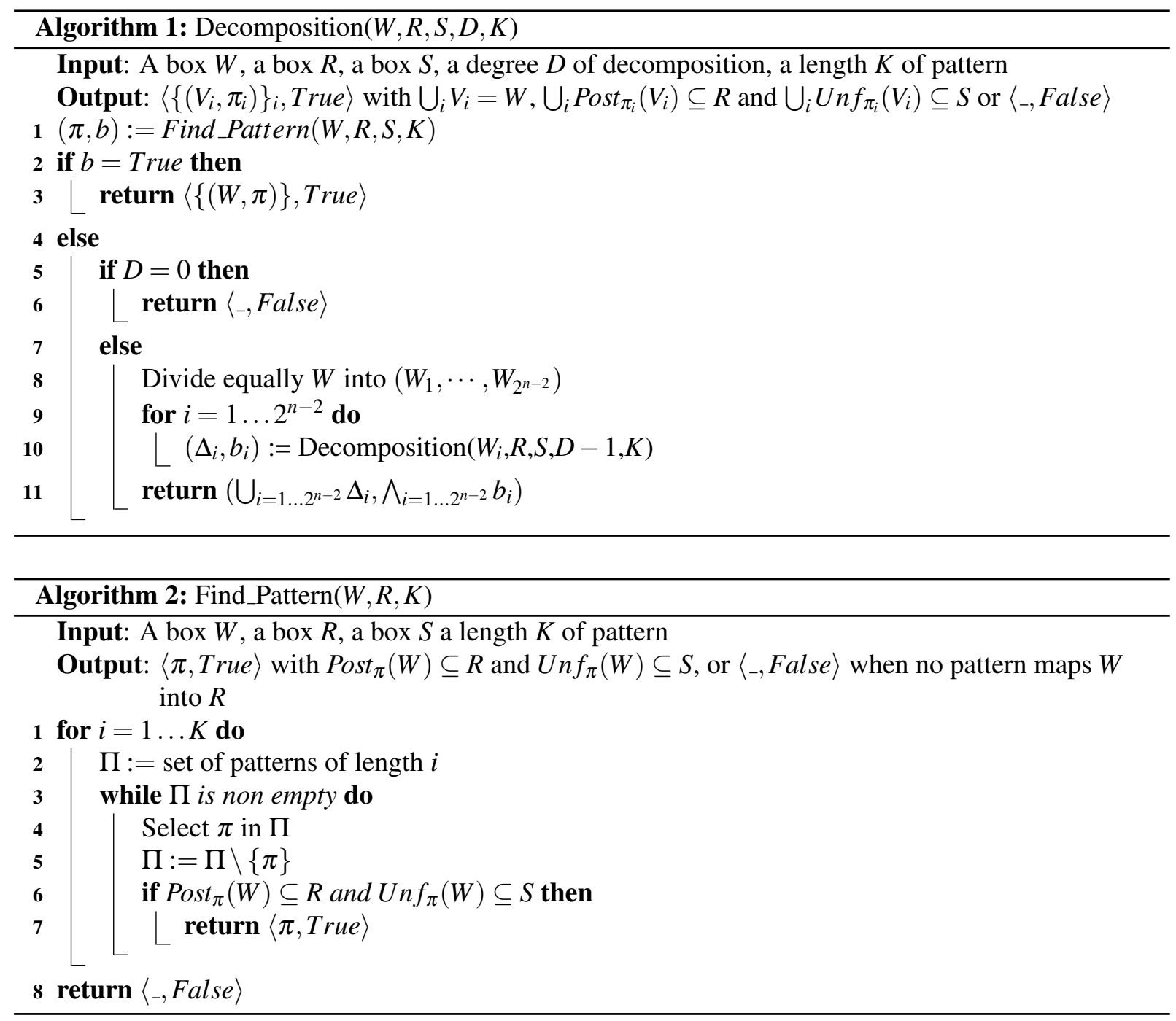

value ( 0 or 1$)$ of the switching cells, i.e., by the value of vector $S=\left[S_{1} S_{2} S_{3} S_{4}\right]^{T}$, 1 There are thus $2^{4}=16$ modes. A mode $S$ induces an output voltage $v_{o}$ of value $\Sigma_{j=1}^{3}\left(S_{j+1}-S_{j}\right) v_{j}+S_{1} v_{\text {high }}-\left(1-S_{1}\right) v_{\text {low }}$, where $v_{\text {low }}$ and $v_{\text {high }}$ are the input voltages of low level and high level respectively. For the sake of simplicity, we suppose: $v_{\text {high }}=v_{\text {low }}=v_{\text {input }}$. The system then outputs 5 different levels of voltage which go from $-v_{\text {input }}$

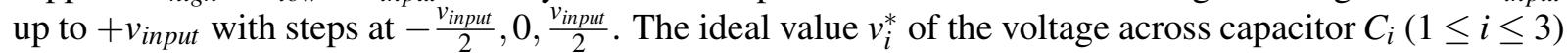
depends on the values of $v_{\text {input }}$. Here we use: $v_{\text {input }}=100 \mathrm{~V}$, and $v_{1}^{*}=150 \mathrm{~V}, v_{2}^{*}=100 \mathrm{~V}, v_{3}^{*}=50 \mathrm{~V}$. The 5 -level converter can be seen as a switched system. Given a mode $S$, the associated dynamics is of the form $\dot{x}(t)=A_{S} x(t)+b_{S}$ with:

$$
A_{S}=\left(\begin{array}{cccc}
-\frac{1}{R_{1} C_{1}} & 0 & 0 & \frac{S_{1}-S_{2}}{C_{1}} \\
0 & -\frac{1}{R_{2} C_{2}} & 0 & \frac{S_{2}-S_{3}}{C_{2}} \\
0 & 0 & -\frac{1}{R_{3} C_{3}} & \frac{S_{3}-S_{4}}{C_{3}} \\
\frac{S_{2}-S_{1}}{L_{\text {Load }}} & \frac{S_{3}-S_{2}}{L_{\text {Load }}} & \frac{S_{4}-S_{3}}{L_{\text {Load }}} & -\frac{R_{\text {Load }}}{L_{\text {Load }}}
\end{array}\right) \text { and } b_{S}=\left(\begin{array}{c}
0 \\
0 \\
0 \\
\frac{\left(2 S_{1}-1\right) v_{\text {input }}}{L_{\text {Load }}}
\end{array}\right)
$$

By controlling the modes at each sampling time, one can synthesize a 5-level staircase function. Not all the transitions between modes are admissible: we allow to switch only one (pair of) cell(s) at a time.

${ }^{1}$ Besides, we have: $S_{5}=\neg S_{1}, S_{6}=\neg S_{2}, S_{7}=\neg S_{3}$ and $S_{8}=\neg S_{4}$. 
The graph of admissible transitions during a cycle is depicted in Figure 3 . The nodes of the graph are labeled by the modes. Each path represents a possible pattern for one cycle, leading from voltage $-v_{\text {input }}$ (mode 0000$)$ to voltage $+v_{\text {input }}$ (mode 1111$)$ through voltages $-\frac{v_{\text {input }}}{2}, 0, \frac{v_{\text {input }}}{2}$ then back to voltage $-v_{\text {input }}$ (mode 0000) through voltages $\frac{v_{\text {input }}}{2}, 0, \frac{v_{\text {input }}}{2}$. There are thus 576 possible patterns for generating a 5-level staircase signal on one cycle.

We explain in the following how to apply the tool MINIMATOR in order to find a safe decomposition involving these patterns.

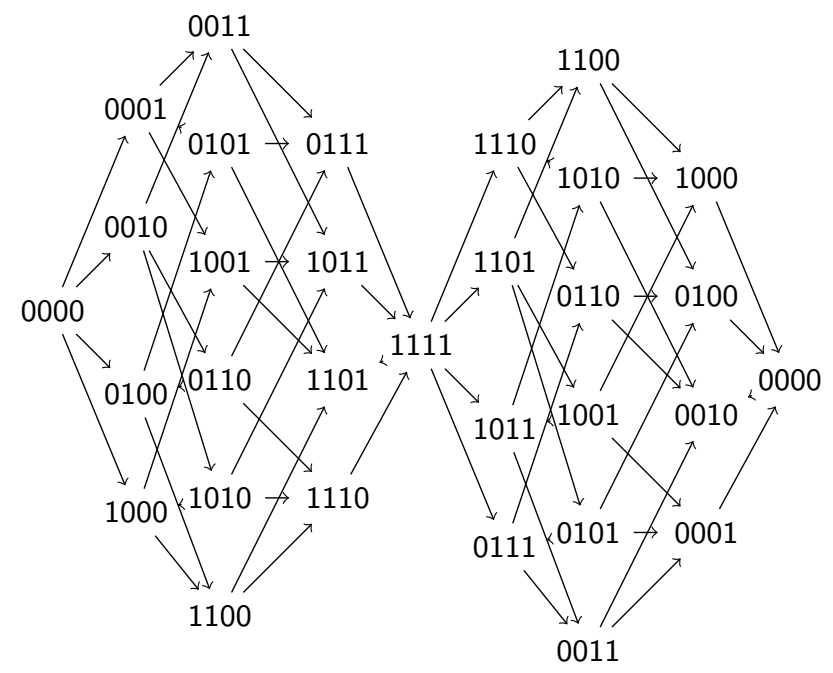

Figure 3: Transition graph corresponding to a cycle of 5-level staircase signal

\subsection{Application of the Decomposition procedure to a 5-level converter}

We consider the following numerical values of the electrical parameters: $v_{\text {input }}=100 \mathrm{~V}, R_{\text {Load }}=50 \Omega$, $C_{1}=C_{2}=C_{3}=0.0012 \mathrm{~F}, L_{\text {Load }}=0.2 \mathrm{H}, R_{1}=R_{2}=R_{3}=20,000 \Omega, T=8 \tau=0.02 s$ (which corresponds to a frequency of $50 \mathrm{~Hz}$ ).

In this context, a 5-level converter outputs ideally a staircase waveform with an amplitude of $200 \mathrm{~V}$, centered around $0 \mathrm{~V}$. We consider that a variation of $\pm 5 \mathrm{~V}$ is admissible as it represents a variation of $10 \%$ on the least charged capacitor $C_{3}$. It is interesting to notice that at each beginning of a cycle the value of $i$ is null. This suggest to look for a state-dependent control which depends only on the capacitor voltages $v_{1}, v_{2}, v_{2}$, and not on the value of $i$. We will thus focus on the voltage dimensions of the control box $R$ and disregard its intensity dimension. For $R$, we take $R=[145,155] \times[95,105] \times[45,55]$, which corresponds to a product of intervals centered around the ideal values with a variation of $\pm 5 \mathrm{~V}$ (i.e., $10 \%$ of the least charged capacitor $C_{3}$ ). For $S$, we take $R+\varepsilon$ with $\varepsilon=1 V$, which means that we have an additional tolerance of $\pm 1 V$ for the fluctuations occurring between two beginnings of cycle.

Given $R=[145,155] \times[95,105] \times[45,55]$ and $S=[144,156] \times[94,106] \times[44,56]$, we perform the procedure of Decomposition, implemented in MINIMATOR tool, on a machine equipped with an Intel core 2 CPU X6800 at $2.93 \mathrm{GHz}$ and with $2 \mathrm{GiB}$ of Ram memory. With parameters $d=1$ and $k=8$, the procedure outputs in 60 seconds a decomposition $\Delta=\left\{\left(V_{i}, \pi_{i}\right)\right\}_{i=1, \ldots, 8}$ with:

- $V_{1}=[145,150] \times[95,100] \times[45,50]$

- $V_{2}=[145,150] \times[95,100] \times[50,55]$

- $V_{3}=[145,150] \times[100,105] \times[45,50]$ 
- $V_{4}=[145,150] \times[100,105] \times[50,55]$

- $V_{5}=[150,155] \times[95,100] \times[45,50]$

- $V_{6}=[150,155] \times[95,100] \times[50,55]$

- $V_{7}=[150,155] \times[100,105] \times[45,50]$

- $V_{8}=[150,155] \times[100,105] \times[50,55]$

and

- $\pi_{1}:(0000 \rightarrow 0001 \rightarrow 0101 \rightarrow 1101 \rightarrow 1111 \rightarrow 1101 \rightarrow 0101 \rightarrow 0001 \rightarrow 0000)$

- $\pi_{2}:(0000 \rightarrow 0100 \rightarrow 0101 \rightarrow 1101 \rightarrow 1111 \rightarrow 1101 \rightarrow 0101 \rightarrow 0100 \rightarrow 0000)$

- $\pi_{3}:(0000 \rightarrow 0001 \rightarrow 0011 \rightarrow 1011 \rightarrow 1111 \rightarrow 1011 \rightarrow 0011 \rightarrow 0001 \rightarrow 0000)$

- $\pi_{4}:(0000 \rightarrow 0010 \rightarrow 0011 \rightarrow 1011 \rightarrow 1111 \rightarrow 1011 \rightarrow 0011 \rightarrow 0010 \rightarrow 0000)$

- $\pi_{5}:(0000 \rightarrow 1000 \rightarrow 1010 \rightarrow 1110 \rightarrow 1111 \rightarrow 1110 \rightarrow 1010 \rightarrow 1000 \rightarrow 0000)$

- $\pi_{6}:(0000 \rightarrow 1000 \rightarrow 1100 \rightarrow 1101 \rightarrow 1111 \rightarrow 1101 \rightarrow 1100 \rightarrow 1000 \rightarrow 0000)$

- $\pi_{7}:(0000 \rightarrow 0100 \rightarrow 0110 \rightarrow 0111 \rightarrow 1111 \rightarrow 0111 \rightarrow 0110 \rightarrow 0100 \rightarrow 0000)$

- $\pi_{8}:(0000 \rightarrow 1000 \rightarrow 1010 \rightarrow 1011 \rightarrow 1111 \rightarrow 1011 \rightarrow 1010 \rightarrow 1000 \rightarrow 0000)$

By Proposition 1, the control of $R$ induced by $\Delta$ is safe w.r.t. $S$ : under the control induced by $\Delta$, all the trajectories starting from $R$ always stay in $S$. This guarantees that the property of capacitor voltage balance is satisfied. We present in Figures 4 and 5 a numerical simulation of this controller on the system starting from the point $v_{1}(0)=150 \mathrm{~V}, v_{2}(0)=100 \mathrm{~V}, v_{3}(0)=50 \mathrm{~V}$ and $i(0)=-3 \mathrm{~A}$. This simulation has been performed using tool PLECS [11]. One can check on the simulation that the system state always stays inside $S$.

\subsection{Application of the Decomposition procedure to a 7-level converter}

We now consider the case of an $\ell$-level converter with $\ell=7$. There are now 6 pairs of switching cells

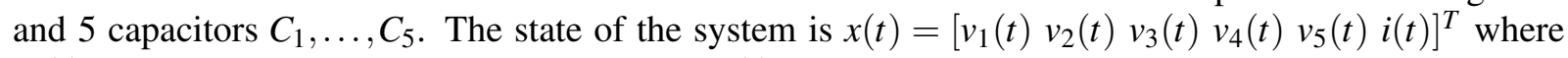
$v_{j}(t)$ is the voltage across $C_{j}(1 \leq j \leq 5)$ and $i(t)$ is the current flowing in the circuit. The generated waveform now goes from $-v_{\text {input }}$ up to $+v_{\text {input }}$ with steps at $-\frac{2}{3} v_{\text {input }},-\frac{1}{3} v_{\text {input }}, 0, \frac{1}{3} v_{\text {input }}, \frac{2}{3} v_{\text {input }}$, and the cycle duration is $T=12 \tau$. There are now 518,400 possible patterns for generating an 7-level staircase signal on 1 cycle. We used the following values for the system constants: output at $50 \mathrm{~Hz}{ }^{2}$ capacitances of $0.1 F$, resistor values $50 \Omega$, inductor values $0.137 \mathrm{H}, v_{\text {input }}=300 \mathrm{~V}$. Ideally, the output is thus a staircase waveform with an amplitude of $600 \mathrm{~V}$, centered around $0 \mathrm{~V}$, and the ideal values $v_{i}^{*}$ of the capacitor voltages of the capacitor $C_{i}$ are given by: $v_{1}^{*}=500 \mathrm{~V}, v_{2}^{*}=400 \mathrm{~V}, v_{3}^{*}=300 \mathrm{~V}, v_{4}^{*}=200 \mathrm{~V}, v_{5}^{*}=100 \mathrm{~V}$. For $R$, we take $R=[495,505] \times[395,405] \times[295,305] \times[195,205] \times[95,105]$, which corresponds to a product of intervals centered around the ideal values with a variation of $\pm 5 \mathrm{~V}$ (i.e., $5 \%$ of the least charged capacitor $C_{5}$ ). For $S$, we take $R+\varepsilon$ with $\varepsilon=1 V$, which means that we have an additional tolerance of $\pm 1 V$ for the fluctuations occurring between two beginnings of cycle. On the same machine as in Section 3.2, with parameters $d=1$ and $k=12$, MINIMATOR outputs in 98 minutes a decomposition $\Delta$ which is safe w.r.t. $S$. See [3] for more details.

We present in Figures 6 and 7 a numerical simulation of the controlled system starting from the point $v_{1}(0)=500 \mathrm{~V}, v_{2}(0)=400 \mathrm{~V}, v_{3}(0)=300 \mathrm{~V}, v_{4}(0)=200 \mathrm{~V}, v_{5}(0)=100 \mathrm{~V}$ and $i(0)=-2.5 \mathrm{~A}$. One can check again on the simulation that the system state always stays inside $S$.

It is difficult to perform experiments with $\ell$ greater than 7 with the present implementation. The complexity of the state decomposition procedure is indeed exponential in the number $\ell$ of levels. We are presently implementing MINIMATOR on a parallel computing architecture (see [9]) in order to increase the tractable number of levels.

\footnotetext{
${ }^{2}$ which corresponds to $T=12 \tau=0.02 \mathrm{~s}$
} 


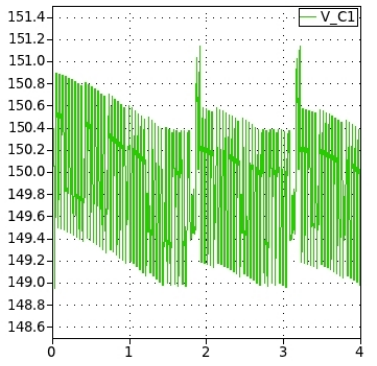

(a) Voltage $v_{1}=f(t)$

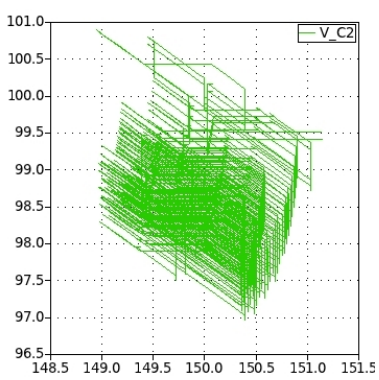

(d) Projection in plane $\left(v_{1}, v_{2}\right)$

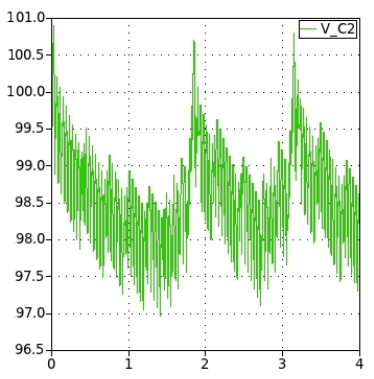

(b) Voltage $v_{2}=f(t)$

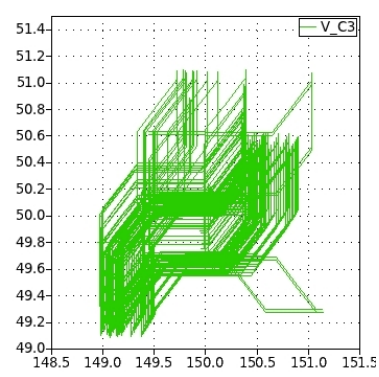

e) Projection in plane $\left(v_{1}, v_{3}\right)$

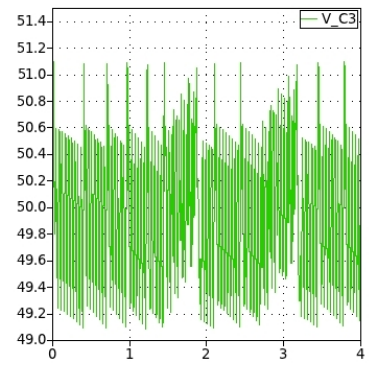

(c) Voltage $v_{3}=f(t)$

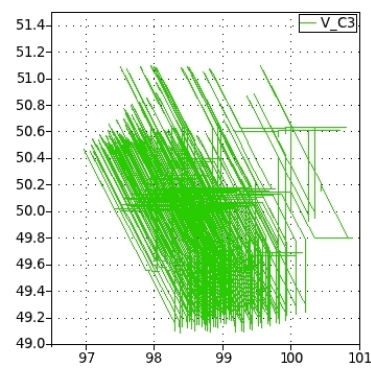

(f) Projection in plane $\left(v_{2}, v_{3}\right)$

Figure 4: Capacitor voltages

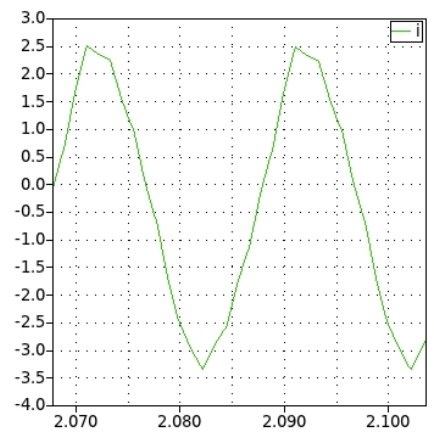

(a) Current $i$

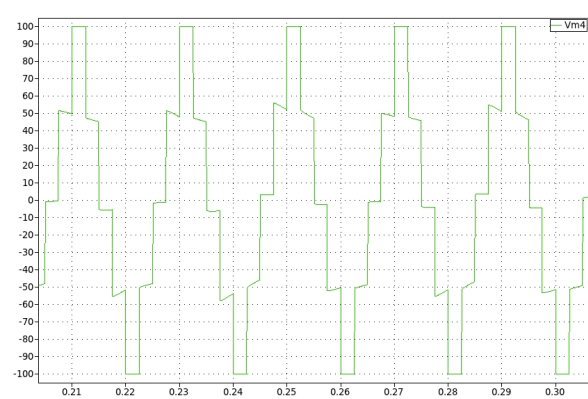

(b) Output voltage $v_{o}$

Figure 5: Current and output voltage

\section{Physical Experimentations on a 5-level Converter}

A prototype of the 5-level flying capacitor has been realized by the SATIE Laboratory in order to test our control strategy on an actual system. See Figure 8 for a picture of the prototype. Our control strategy was applied to the system via Simulink and a dSpace ${ }^{\circledR}$ interface. The results are presented in Figure 9 for the output voltage and the capacitor charges. In Figure 10, we present the same results but with a larger scale on the capacitor voltage to see the fluctuations around the reference values. As we can see, the experimental results are very closed to those obtained by simulation with PLECS of Section 3.2. In Figure 11, we represent the output voltage together with the current (after appropriate resizing) flowing the load. During the experimentations, we have successfully tested the robustness of the controller in presence of the following perturbations: 

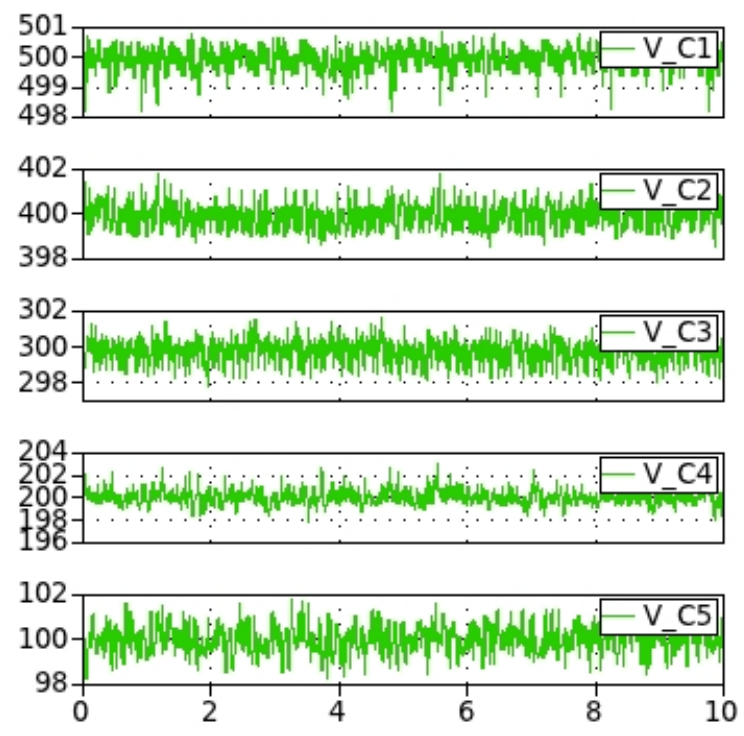

Figure 6: Capacitor voltages

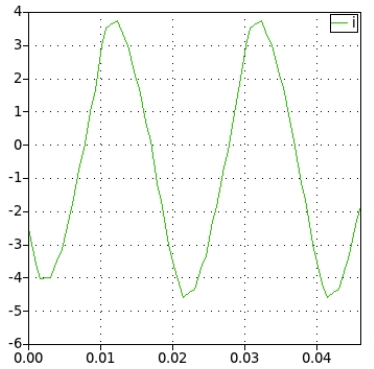

(a) Current $i$

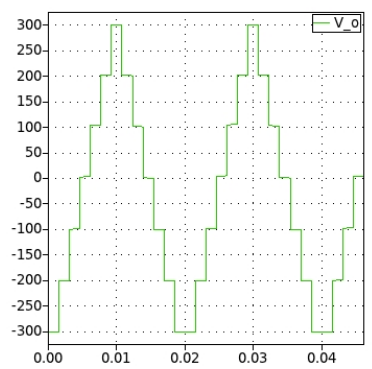

(b) Voltage $v_{o}$

Figure 7: Current and output voltage

1. The ideal voltage source as input is no longer ideal but its values fluctuate around the reference value.

2. We use a time-varying period $T$ of cycle (instead of a constant one), and check the preservation of the capacitor voltages balance. The result of this experiment is depicted in Figure 12 .

Although these preliminary tests of robustness are promising, they need to be consolidated, in particular in presence of significant variations of resistor loads.

\section{Final Remarks}

We have synthesized a control strategy for a 5-level and a 7-level flying capacitor converters using the method of state space decomposition. This control is state-dependent and is interesting because:

- at each electrical cycle, the controller indicates all the subsequent switching modes needed to produce one period of the output voltage (instead of just the next switching mode),

- the controller takes into account only the capacitor voltages state and not the intensity state; this is interesting because for practical applications, a current sensor is not always desired (see [2]). 


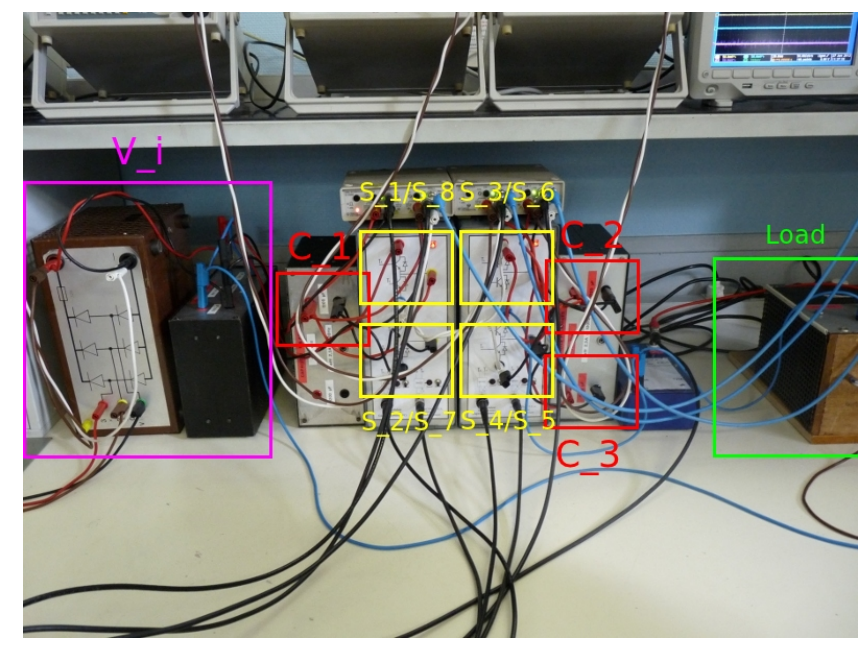

Figure 8: Prototype built by SATIE

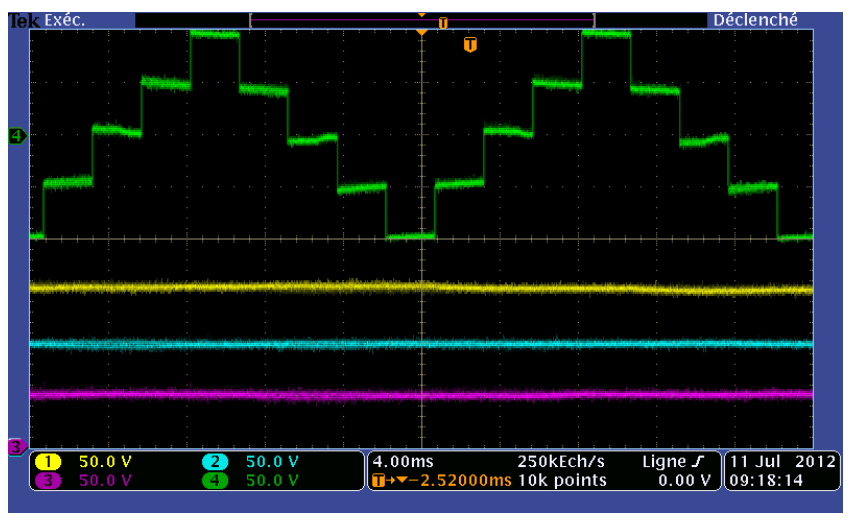

Figure 9: Output voltage (above in green) and capacitor voltages (below)

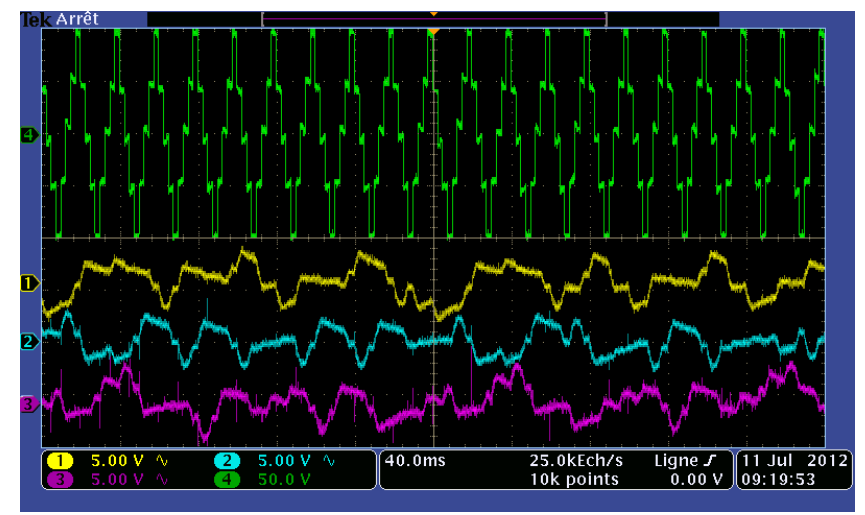

Figure 10: Zoom of output voltage (above) and capacitors voltages (below)

We have checked by numerical simulations and physical experimentations that the control satisfies the capacitor voltage balancing and the staircase shape of the output voltage. We have also checked the robustness of the method with respect to several sources of perturbation. 


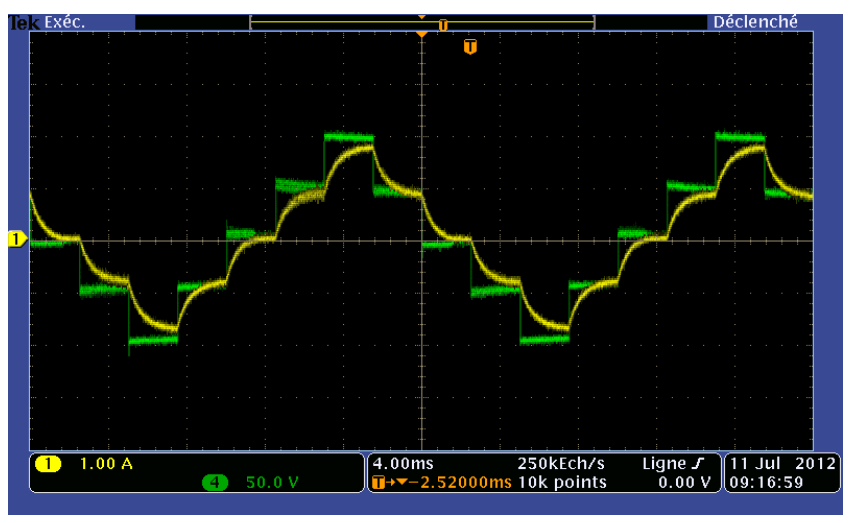

Figure 11: Output voltage (in green) and current (in yellow, after appropriate resizing) in the circuit

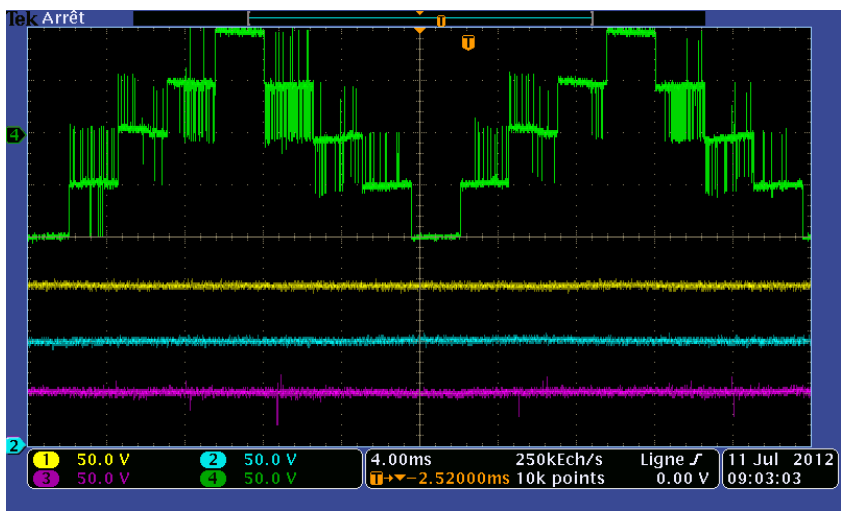

Figure 12: Output voltage (above) and capacitor voltages (below) in presence of time-varying period $T$

The method can be easily refined in order to generate sinusoidal-like output signals rather than the triangular-like output signals generated here: it suffices to adjust the switching instants within the period $T$ of the cycle, instead of using uniformly $\tau$.

The method can be applied in principle to any number of levels for the flying capacitor topology. However, it suffers from an exponential increase of complexity when the level $\ell$ grows: the method reaches its limit for $\ell=9$, which corresponds to a dimension $n=7$ of the state space.

For $\ell=5,7$, the Decomposition procedure is well-suited to the flying capacitor topology: the pattern length input $k$ is $2 \times(\ell-1)$ where $\ell$ is the number of levels of the converter, and the depth input $d$ is 1 , which means that the decomposition is found after a single bisection. Note however that such simple decompositions of the state space do not necessarily exist for other topologies: for multilevel modular converter toplogy [7], we had to propose in [13] a different and specialized algorithm which takes additionally into account the value of the intensity state.

In future work, we plan to improve the robustness of the decomposition method for flying capacitor topology under variations of the resistive and inductive load. This will allow us to model the time-varying load of electrical networks, which is a basic feature of electricity distribution, and a major challenge today for renewable-energy technologies. We are also implementing the tool MINIMATOR on a parallel computing architecture in order to synthesize correct-by-design controls for multilevel converters with a greater numbe $\ell$ of levels.

Acknowledgement. We are grateful to Stéphane Lefebvre for numerous helpful discussions. We also thank the anonymous referees for their constructive comments. This work has been done within the 
framework of projects BOOST and BOOST2 supported by Institut Farman.

\section{References}

[1] I. Cervantes, F.J. Perez-Pinal \& A. Mendoza-Torres (2009): Hybrid Control of DC-DC Power Converters. In: Renewable Energy (Chapter 10), T J Hammons, pp. 173,193, doi $10.5772 / 7370$

[2] Zhong Du, L.M. Tolbert, B. Ozpineci \& J.N. Chiasson (2009): Fundamental Frequency Switching Strategies of a Seven-Level Hybrid Cascaded H-Bridge Multilevel Inverter. IEEE Transactions on Power Electronics 24(1), pp. 25-33, doi 10.1109/TPEL.2008.2006678.

[3] G. Feld, L. Fribourg, D. Labrousse, B. Revol \& R. Soulat (2012): Correct by design control of 5-level and 7level converters. Research Report LSV-12-25, Laboratoire Spécification et Vérification, ENS Cachan, France.

[4] Laurent Fribourg \& Romain Soulat (2013): Stability Controllers for Sampled Switched Systems. In Parosh Aziz Abdulla \& Igor Potapov, editors: Proceedings of the 7th Workshop on Reachability Problems in Computational Models (RP'13), Lecture Notes in Computer Science 8169, Springer, Uppsala, Sweden, pp. 135-145, doi:10.1007/978-3-642-41036-9_13.

[5] A. Girard, G. Pola \& P. Tabuada (2010): Approximately Bisimilar Symbolic Models for Incrementally Stable Switched Systems. IEEE Trans. on Automatic Control 55, pp. 116-126, doi:10.1109/TAC.2009.2034922.

[6] W. Kühn (1998): Zonotope dynamics in numerical quality control. Mathematical Visualization, pp. 125-134, doi:10.1007/978-3-662-03567-2_10

[7] A. Lesnicar \& R. Marquardt (2003): An innovative modular multilevel converter topology suitable for a wide power range. In: Power Tech Conference Proceedings, 2003 IEEE Bologna, 3, pp. 6 pp. Vol.3-, doi $10.1109 /$ PTC.2003.1304403

[8] T.A. Meynard \& H. Foch (1992): Multi-level conversion: high voltage choppers and voltage-source inverters. In: 23rd Annual IEEE Power Electronics Specialists Conference (PESC '92), 1, pp. 397-403, doi $10.1109 /$ PESC.1992.254717

[9] MINIMATOR Web Page. https://bitbucket.org/ukuehne/minimator/.

[10] Octave Web Page. http://www.gnu.org/software/octave/.

[11] PLECS Web Page. http://www.plexim.com.

[12] B. Singh, N. Mittal, K.S. Verma, D. Singh, S.P. Singh, R. Dixit, M. Singh \& A. Baranwal (2012): Multilevel inverter: A literature survey on topologies and control strategies. International Journal of Reviews in Computing 10.

[13] Romain Soulat, Guillaume Hérault, Denis Labrousse, Bertrand Revol, Gilles Feld, Stéphane Lefebvre \& Laurent Fribourg (2013): Use of a full wave correct-by-design command to control a multilevel modular converter. In Philippe Lataire, editor: Proceedings of the 15th European Conference on Power Electronics and Applications (EPE'13), IEEE Power Electronics Society, Lille, France, pp. 1,8, doi:10.1109/EPE.2013.6634448. Available at http://www.lsv.ens-cachan.fr/Publis/PAPERS/PDF/SHLRFLF-epe13.pdf.

[14] Paulo Tabuada (2009): Verification and Control of Hybrid Systems: A Symbolic Approach. Springer Publishing Company, Incorporated, doi $10.1007 / 978-1-4419-0224-5$. 